\title{
XVI Congreso Nacional de la Sociedad Española de Educación Médica (SEDEM)
}

Fiel a su cita bienal, la Sociedad Española de Educación Médica convoca a todos los educadores de las ciencias de la salud de nuestro país a su Congreso Nacional, en su decimosexta edición. En esta ocasión quiero destacar que, por primera vez en sus 33 años de historia, la SEDEM celebra su congreso en un marco distinto al de una Facultad de Medicina.

La SEDEM, a lo largo de su historia, ha celebrado siempre sus congresos en una Facultad de Medicina, en las cuales siempre se ha sentido acogida. Ello es lógico habida cuenta que la Sociedad se fundó en una institución universitaria, que sus fundadores eran fundamentalmente profesores de universidad, que un gran número de sus socios son profesores y que siempre, como en esta ocasión, se ha tenido el inestimable apoyo de la Conferencia Nacional de Decanos de Facultades de Medicina Españolas.

En los últimos diez años se han sucedido importantes cambios en las estructuras de los sistemas sanitarios y la SEDEM, sensible a estos procesos, se ha acercado progresivamente al mundo de las profesiones sanitarias, en todas sus etapas, formación de pregrado, formación de postgrado y el desarrollo profesional continuo o formación continuada. En este sentido, un factor facilitador del acercamiento a otros ámbitos diferentes del pregrado fue la firma en el año 2001 de un convenio marco de colaboración entre la SEDEM y el Instituto de Salud Carlos III. Este convenio estimulaba la celebración de actividades conjuntas, entre las que cabe destacar los Cursos de Formación de Profesorado de las Licenciaturas y Diplomaturas en Ciencias de la Salud y Cursos de Formación de Tutores en Postgrado, organizados por el Departamento de Metodología Docente y Gestión de la Educación en Ciencias de la Salud del ISCIII, y a los cuales la SEDEM ha aportado su soporte científico y sus recursos humanos. Precisamente a la luz de dicho convenio surgió la idea de aprovechar los recursos del Instituto de Salud Carlos III para organizar la XVI edición del Congreso de SEDEM, dando un nuevo empuje al referido proceso aproximación de la Sociedad al mundo de la sanidad. Así pues, hemos de contemplar este XVI Congreso de la SEDEM como un nuevo fruto de esta fecunda colaboración. Desde este editorial, la Sociedad Española de Educación Médica y su Junta Directiva quieren expresar su agradecimiento y reconocimiento al Instituto de Salud Carlos III por la disponibilidad y la colaboración de todo su personal en la organización del congreso, y muy en especial a su Director, el Prof. Antonio Campos Muñoz, que siempre fue un entusiasta convencido de esta colaboración.

El lema escogido para esta ocasión y que intenta ser el denominador común del Congreso ha sido el de la Calidad en Educación Médica. La calidad en la educación en las Ciencias de la Salud es la mejor garantía para que nuestros profesionales sean competentes. Conseguir la calidad pasa, inexcusablemente, por asegurar la calidad tanto de nuestras instituciones docentes como del profesorado. Por ello el Congreso se inicia con una lección magistral a cargo de la Profa. Miriam Friedman Ben-David sobre la relación entre competencias profesionales y educación y se continua con cuatro ponencias relacionadas con el lema del congreso. Una de ellas está dedicada a la evaluación y acreditación de las Instituciones docentes. La cultura de la evaluación y de la acreditación empieza a calar fuertemente en nuestro país, como lo demuestra el establecimiento de Agencias de Evaluación de Calidad y Acreditación a nivel estatal y autonómico, siendo cada vez más las instituciones evaluadas. Por ello esta ponencia, a cargo de expertos nacionales e internacionales, discutirá los procesos de evaluación y acreditación institucional, repasando las experiencias mundiales, europeas, estatales y autonómicas, y el impacto de la evaluación en la calidad de la institución. Y todo ello tomando como referencia estándares internacionales de calidad como los elaborados por la WFME y el IIME, representado en el congreso y en esta ponencia por su director, un gran amigo de la SEDEM el Prof. A. Wojtcak.

Una segunda ponencia, a cargo de expertos nacionales, tratará los fines de la evaluación del profesorado y los procedimientos y estrategias de evaluación. Seguro que pondrán en evidencia que la evaluación objetiva de la actividad docente del profesorado no sólo es necesaria sino posible.

Una tercera ponencia estará dedicada al Tutor de Postgrado. En ésta se tratarán aspectos relacionados con la acreditación, el reconocimiento y la evaluación de los mismos y de los residentes. Finamente, la cuarta ponencia estará dedicada de forma específica a la enseñanza y aprendizaje de la Salud Pública, abordando las necesidades de formación de estos profesionales, el papel de las Escuelas de Salud Pública, y la definición del perfil profesional. Asimismo, la Conferencia Nacional de Decanos de 
Facultades de Medicina españolas tratará sobre cómo afecta el proceso de convergencia europea a las Facultades.

Además, el congreso celebrará varias sesiones temáticas dedicadas a los Contenidos, Metodología y Entornos docentes de la Medicina Familiar y Comunitaria, a la Gestión de la docencia en los hospitales universitarios y a la Biblioteca Nacional de Ciencias de la Salud. Como no podría ser de otra manera, por ser una de las actividades más importantes del congreso, dedicaremos espacio y tiempo relevantes a la presentación de comunicaciones libres en forma oral o de panel. Finalmente, también se podrá participar en los diferentes talleres programados al efecto.

Como viene siendo ya habitual desde el XIV Congreso celebrado en Lleida, las ponencias y comunicaciones aparecen publicadas en la revista Educación Médica de la cual la SEDEM es auspiciadora. Sin duda, desde que esto es así, la participación tanto en número como en calidad de las aportaciones a los congresos han aumentado de forma significativa, ya que la revista supone el primer órgano de difusión en lengua castellana de los trabajos y estudios de educación médica y de ciencias de la salud. Por ello la SEDEM reconoce y agradece el esfuerzo realizado por la Fundación Educación Médica, actual responsable de la publicación. Este año existe además otro motivo de satisfacción. Junto con el ejemplar del congreso se distribuye un suplemento especial que contiene tres documentos que consideramos han de ser muy útiles para todos los educadores. Este suplemento especial en cuya preparación han participado activamente miembros de la Sociedad, contiene por un lado dos documentos que hacen referencia a las competencias esenciales que caracterizan la profesión médica y que debiera constituir los requisitos mínimos del producto educativo de cualquier Facultad de Medicina.

Los educadores relacionados con las ciencias de la salud estamos necesitados de un lenguaje común y de un cuerpo de conceptos compartido por todos, que permita un entendimiento entre todos aquellos que trabajan en este campo científico. Por ello, el tercer documento que se presenta en al suplemento, el glosario de términos elaborado por el Institute for International Medical Education, será de gran importancia para todos los educadores. Estamos seguros de que los tres documentos que reúne este suplemento tendrán una muy buena acogida por parte de todos.

Finalizo dando la bienvenida a todos los participantes en este XVI Congreso y quiero agradecer especialmente la presencia de aquellos que han viajado desde el vecino Portugal y de aquellos que lo han hecho desde diferentes países de Américalatina. A todos os deseo que el Congreso satisfaga vuestros objetivos.

$\mathrm{Al}$ ser este mi primer congreso en el que ocupo la presidencia de SEDEM, confío que esté a la altura de los anteriores presididos por la Profa. Margarita Barón, que sigue liderando la Educación Médica desde la Presidencia de AMEE, para la que acaba de ser reelegida por tres años más. Deseo que sus éxitos en AMEE sean tantos como lo fueron en nuestra Sociedad Española de Educación Médica, y que las sociedades científicas de educación médica sigan liderando el proceso de mejora y garantía de calidad en todas la etapas del continuum de la formación médica.

Jorge Palés Argullós 